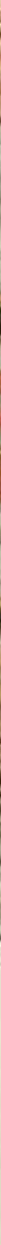

\title{
Wand- und Deckenmalereien in Torgau vom 15. bis zum 17. Jahrhundert
}

\author{
Angelica Dülberg
}

Obwohl Wand- und Deckenmalereien seit ihrer Entstehungszeit stets von gravierenden Zerfallserscheinungen bedroht und vielfältigen Umwelteinflüssen ausgesetzt waren sowie gleichzeitig in den folgenden Jahrhunderten nicht mehr dem Zeitgeschmack entsprachen und Umbauten zum Opfer fielen, gehört Torgau neben Freiberg, Meißen, Pirna und Görlitz zu den sächsischen Städten, die noch eine beachtliche Anzahl dieser Kunstgattung in den Bürgerhäusern aus der Zeit um 1500 bis zum Anfang des 17. Jahrhunderts aufweisen. Dies ist dem Umstand zu verdanken, dass Wandmalereien oft lediglich übertüncht oder mit zeitgemäßen Darstellungen übermalt und später - im 19. Jahrhundert - mit Tapeten überklebt wurden. So wurden sie in gewisser Weise konserviert und kommen nach mühsamen Freilegungsarbeiten heute wieder original, jedoch mehr oder weniger fragmentarisch zum Vorschein. Häufiger und besser erhalten sind die bemalten Holzbalkendecken, weil es seit dem Barock häufig üblich war, darunter niedrigere Stuckdecken einzuziehen. Damit zerstörte man die originalen Malereien nicht, sondern konservierte sie eher, so dass nach der Entfernung der oftmals nicht allzu wertvollen Stuckdecken die farbliche Pracht der Malereien des 16. und beginnenden 17. Jahrhunderts verhältnismäßig gut wieder zutage getreten ist. ${ }^{1}$

Wohl die frühesten Wandmalereien sind in dem ehemaligen Kurfürstlichen Kanzleihaus erhalten, in einem im Erdgeschoss östlich der sogenannten „Großen Stube“ liegenden, tonnengewölbten kleinen Raum, der offensichtlich im letzten Viertel des 15. Jahrhunderts gebaut wurde. ${ }^{2}$ Im Inventarium von 1601 werden ,,6 große eisern rincken [Ringe]“ erwähnt, die an der rechten Wand noch erhalten sind, sowie ein großer viereckiger Vogelbauer, ,,1 Vogelbaur groß Vierecket Vonn eisenn vnnd Tratt [Draht]“, der von der Mitte der Decke herabhing. Vermutlich wurden dort die zur Jagd mitgeführten
Torgau, Breite Straße 9, Bürgermeister-Ringenhain-Haus, erstes Obergeschoss, sogenannte „Engelstube“, Deckengemälde mit 44 Engeldarstellungen, 1631 Foto: Claudia Herrmann 
1 Vgl. Angelika Dülberg: Wandund Deckenmalereien vom 15. bis zum ausgehenden 17 . Jahrhundert in Freiberger Bürgerhäusern. Mit einem Katalog der Wand- und Deckenmalereien vom 15 . bis zum 19. Jahrhundert in Freiberg. In: Yves Hoffmann/ Uwe Richter (Hrsg.): Denkmale in Sachsen. Stadt Freiberg. Beiträge. Bd. 3. Freiberg 2004, S. 828-926, hier S. 828-832. Vgl. auch Rolf Gramatzki: Bemalte Holzdecken in der Stadt und im ehemaligen Erzstift/Herzogtum Bremen. Versuch einer typologischen und ikonographischen Bestandsaufnahme. In: Bremisches Jahrbuch 63 (1985), S. 33-91, hier S. 34.

2 Thomas Rau: Zur Baugeschichte des Kanzleihauses. In: Das Kurfürstliche Kanzleihaus zu Torgau. Erkenntnisse zur Bau- und Nutzungsgeschichte. Schriften des Torgauer Geschichtsvereins 2 (2001), S. 11-20, hier S. $13 \mathrm{f}$.

3 Arnim Schneiderheinze: Das Inventarium von 1601. Beobachtungen zur Struktur, Funktionsweise und Ausstattung der Kurfürstlichen Kanzlei und des Torgauer Amtes. In: Das Kurfürstliche Kanzleihaus (wie Anm. 2), S. 83--124, hier S. 92, 100.

4 Vgl. Angelica Dülberg: Wand- und Deckenmalereien in Torgauer Bürgerhäusern und im Schloss Hartenfels. In: Torgau - Stadt der Renaissance. 2. Auflage Dresden 2004, S. 122-135, hier S. 123 f., Abb. 2; Angelica Dülberg: Bemerkungen zu einigen Wand- und Deckenmalereien in Torgauer Bürgerhäusern und im Schloss Hartenfels. In: Lutherstadt Wittenberg, Torgau und der Hausbau im 16. Jahrhundert. In: Jahrbuch für Hausforschung 62 (2015), S. 207222, Abb. 1. Der vorliegende Aufsatz geht leicht überarbeitet und ergänzt auf diese Publikationen zurück.

Torgau, Kurfürstliches Kanzleihaus, Erdgeschoss, sogenanntes "Vogelzimmer", Wandgemälde mit grünem Rankenwerk und einem nackten Mann, Ende des 15. Jahrhunderts Foto: Angelica Dülberg
Jagdvögel gehalten. ${ }^{3}$ Die eisernen Ringe können dazu gedient haben, die erlegten kleineren Tiere wie Rebhühner, Fasanen oder Hasen als Jagdtrophäe aufgehängt zur Schau zu stellen. Dieselbe Funktion hatten große Ringe an der Decke in einem Erdgeschossraum des Schlosses Neuburg an der Donau, an denen große Tiere wie Rotwild oder Wildschweine aufgehängt wurden. Es scheint durchaus möglich, dass die Wandmalereien unmittelbar mit der Funktion des Raumes in Verbindung zu bringen sind.

Den Raum umzieht eine gemalte Brüstung, vor der wechselweise rote und grüne Vorhänge herabhängen, die mit Ringen an einer darüber liegenden Stange befestigt sind. Aus dieser scheint gleichfalls über die gesamte Wand- und Gewölbefläche ein üppiges grünes Rankenwerk emporzuwachsen, in das große Blüten, Früchte und ein Vogel mit langen Schwanzfedern eingewoben sind. Außerdem wurde ein nackter Mann im Rankenwerk entdeckt. ${ }^{4}$ Inzwischen sind, weil kein Geld für weitere Freilegungen zur Verfügung stand, alle Rankenmalereien bis auf eine Musterachse mit dem nackten Mann wieder übertüncht worden.

Der Torgauer Raum steht in der Tradition der sogenannten Grünen Gemächer oder Stuben der zweiten Hälfte des 15. Jahrhunderts, die sich vor allem in Böhmen und der heutigen Slowakei sowie insbesondere auch in Tirol erhalten haben.

Unter den zahlreichen in Tirol überlieferten Rankenmalereien gehören diejenigen im alten Pfarrwidum in Lana zu den frühesten Beispielen um 1430. Sie zeigen Jagddarstellungen sowie einen Beizvogel, der mit der Langfessel an der hohen Reck angebunden ist. ${ }^{5}$ Um 1475 sind in der fürstlichen Burg von Meran Windhunde, die Rotwild und Hasen hetzen, und ein Jäger dargestellt, der einen Hirsch mit seinem Spieß abfängt. Weitere Details der Jagd im Rankenwerk sind ein Jagd-

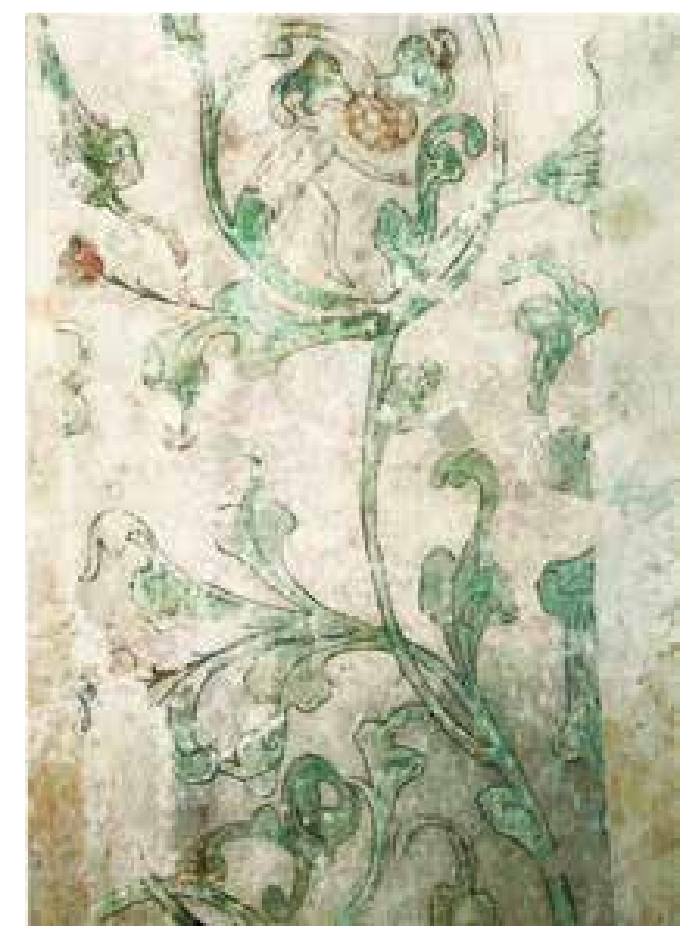

hornbläser mit Hund und der Vogelfang mit Kloben. ${ }^{6}$

Die Hirschjagd ist auch in der Churburg am Ende des 15. Jahrhunderts Hauptthema, oben ein Leithundeführer im Blattwerk, unten eine Hirschjagd. ${ }^{7}$ Im Grünen Saal des Schlosses Reifenstein, geschaffen 1498, klettern kleine, zum Teil mit Pfeilen bewaffnete männliche Wesen in den grünen Ranken, die man jedoch erst auf dem zweiten Blick entdeckt, weil sie farblich ihrer Umgebung angepasst sind. ${ }^{8}$

In dem überaus reich ausgestatteten adligen Ansitz Jöchlsthurn in Sterzing bringen hingegen Blüten, Früchte, Vögel und kleine nackte Menschenkinder starke Farbakzente in die vorwiegend grün monochrome Rankenmalerei auf grünem Grund ein, die durchaus mit derjenigen im Torgauer Vogelzimmer vergleichbar ist. ${ }^{9}$ Auch hier befindet sich unterhalb eine illusionistisch gemalte Sockelzone: An einer Stange sind verschiedenartig gemusterte rote Tücher aufgehängt, die zum Boden hin in Fransen auslaufen.

Bei den genannten Beispielen sind fast immer zwei Realitätsebenen vorhanden: die gemalte Brüstung, über oder an der kostbare, oft gemusterte Teppiche oder Tücher hängen, und darüber die grünen Rankenmalereien, die zum Hauptmotiv geworden sind. Sie drücken ein neues Naturgefühl aus, sollten einerseits den Charakter einer Laube vermitteln oder wurden andererseits im Sinne einer Landschaftsabstraktion als Wald verstanden, wie der Auftrag Kaiser Maximilians I. an Jörg Kölderer deutlich zeigt. ${ }^{10}$ Er sollte die große Stube der Burg zu Innsbruck „mit lauter pamen und wäldern, auch mit Jeger und gefugel“ und im Wappenhaus der Hofrüstkammer „die pretter, deren die gehurn hangen gruen zu malen mit Pamen, vogeln und klainen jägerlein auf das hubischst" bemalen. Das mittelhochdeutsche Wort „pamen“ - „Bäume“ - steht als Synonym für die Ranken und bezeichnet somit direkt ihren vollgültigen Realitätsgrad. Sie werden nicht nur mit Blüten und Früchten belebt, sondern auch mit Vögeln, Wildweibchen, kleinen Jägern und Jagdszenen. Der enge Bezug der Jagd zum Wald ist offenkundig. Es scheint durchaus möglich, dass die Rankenmalereien im Torgauer Vogelzimmer bewusst für den Aufbewahrungsraum der Jagdvögel bezweckt waren, bedenkt man die große Wertschätzung, die die kostbaren Vögel an den europäischen Höfen genossen haben. So ist zum Beispiel überliefert, dass der Herzog von Mailand, Filippo Maria Visconti, für die Pflege seiner Jagdvögel monatlich 3.000 Goldstücke ausgegeben hat.

Bald nach der Ausmalung des „Vogelzimmers“ im Kanzleihaus sind ähnliche Rankenmalereien in dem Torgauer Bürgerhaus Pfarrstraße 3 um 1500 oder am Anfang des 16. Jahrhunderts entstanden. ${ }^{11}$ Über die obere Wandfläche rankt sich grünes Laubwerk, belebt mit bunten Blüten und Früchten, von dem zunächst lediglich an einigen Stellen Reste freigelegt worden waren. Es handelt sich um großes, fleischiges Blattwerk mit differenzierter schwarzer Binnenzeichnung, eingerollten Enden und zarten Blütenstängeln. 


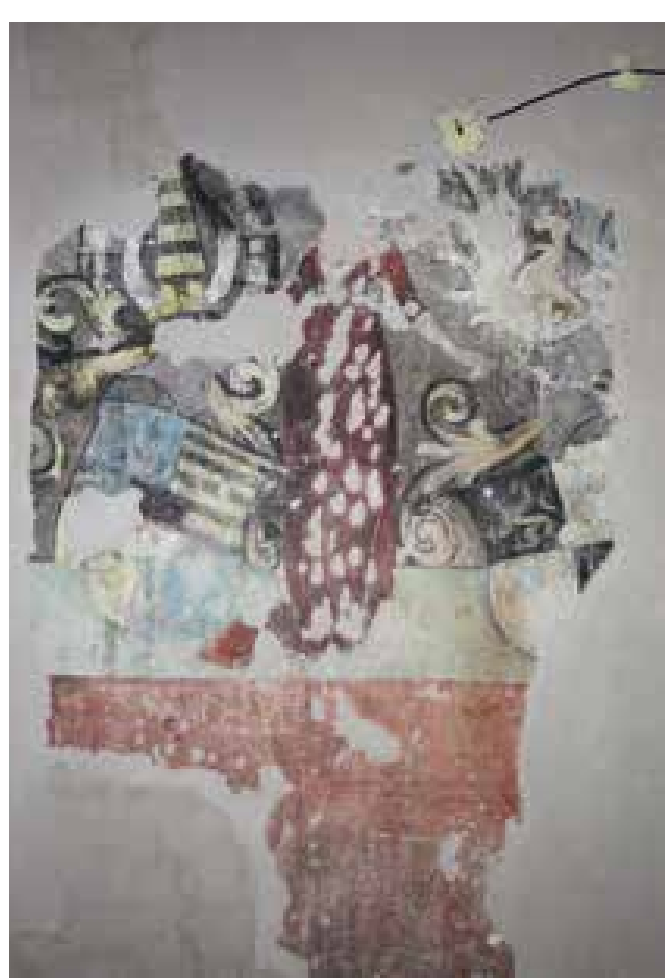

Am Anfang vermutete ich, dass sich ehemals in der unteren Wandzone rund um den Raum eine gemalte rote, profilierte Steinbrüstung entlang gezogen hat, die in den Laibungsflächen der Fenster und an der Südwand bereits belegt war. Jedoch stellte sich später heraus, dass auf der gesamten Wandfläche zwischen den Fenstern von unten grünes Rankenwerk mit großen farbigen Blüten emporrankt, wenngleich das Grün weitgehend vergangen ist. Vermutlich wächst jeweils nahe den Kanten zu den Laibungen der dünne Stamm einer Pflanze in die Höhe, deren Astwerk sich dann über die Wandfläche hin verzweigt.

Auf den grünen Oberkanten der Brüstung stehen schlanke kleine Frauengestalten als Wappenhalterinnen. Sie halten mit ausgebreiteten Armen links und rechts Wappenschilde mit Helmzierden, die als wettinische identifiziert werden konnten. Konkret gehen die Wappendarstellungen auf die Regierungszeit des Kurfürsten Friedrich des Weisen (1486-1525) zurück. ${ }^{12}$

Die Auswahl der Wappen und auch ihre Zusammenstellung in der Pfarrstraße 3 scheinen individuell gewesen zu sein, wie es selbst bei den Wettinern zahlreiche Varianten gegeben hat. Das gevierte Hauptwappen mit dem Herzschild Kursachsens erscheint ein zweites Mal auf der Südwand zur rechten Seite einer Wappenhalterin, die nicht erhalten ist.

Das illusionistische Ausmalungsprogramm wird durch fragmentarische Malereien in der oberen rechten Fensterlaibung ergänzt. Es handelt sich um Reste gemalter roter Gewölberippen und roten Maßwerks. Damit steht der Raum des Hauses Pfarrstraße 3 trotz seines fragmentarischen $\mathrm{Zu}-$ standes in der Reihe weniger erhaltener illusionistisch ausgemalter profaner Räume nördlich der Alpen aus der zweiten Hälfte des 15. und dem Anfang des 16. Jahrhunderts. In Sachsen gehören die Wandmalereien in dem Meißner Haus Rote Stufen 3 sowie vor allem die sogenannte „Görlitzer Schatzkammer" im Haus Untermarkt 5 dazu. ${ }^{13}$ Den Raum umzieht eine gemalte Steinbrüstung, über der kostbare Brokattücher hängen und auf der kostbare Gefäße stehen. Die einfache Spitztonne wird durch ein gemaltes weißgraues Netzrippengewölbe negiert und damit gleichzeitig zu einem fiktiven Rippengewölbe erweitert.

Wie in Görlitz nimmt auch ein Raum des ersten Obergeschosses im Regensburger Haus Obere Bachgasse 8 die vor allem in Kirchen und Kapellen zahlreich nachzuweisenden gemalten fiktiven Rippengewölbe auf. ${ }^{14}$

Vorläufige Quellenstudien und Untersuchungen haben ergeben, dass das Haus vermutlich bis 1535 im Besitz der Familie Reppisch war, wobei Michel Reppisch Torherr und sein Bruder Peter Kammerherr des Kurfürsten waren. Beide sind auch als Bürgermeister von Torgau nachgewiesen. Offensichtlich hat es der Ritter Georg von Minckwitz entgegen anderen Quellen - Lehnsbrief von 1545 - schon 1535 in seinen Besitz übernommen. Dafür spricht eine Ritzung in der Laibung der südlichen Fensteröffnung ,15 M 38 [...] vollendts [...] minckwitz“. Plausibel erscheint es zunächst, dieser alten sächsischen Adelsfamilie, deren Mitglieder höhere Ämter am sächsischen Hofe bekleidet haben als die Familie Reppisch, die anspruchsvolle Ausmalung des Raumes mit den Wappen der Wettiner zuzuweisen. ${ }^{15}$ Dennoch sprechen die beschriebenen Wandmalereien gegen diese späte Datierung. Die Rankenmalereien, die fiktiven Rippenmalereien wie auch die kleinen überlängten Körper der Wappenhalterinnen deuten eher auf eine Entstehungszeit um 1500 hin. Bekräftigt wird die Datierung durch die unmittelbar vergleichbare plastische Darstellung einer Wappenhalterin des Wappenfrieses am südlichen Treppenhaus des Wittenberger Schlosses, die 1493 von Claus Heffner geschaffen wurde und ganz ähnlich einen überlängten Unterkörper mit einem hochgegürteten Gewand zeigt. ${ }^{16}$ Möglicherweise kann sie gar als Vorbild für die Wandmalereien gedient haben, entspricht doch der zur Rechten gehaltene Wappenschild genau dem der Frau im roten Gewand.

Zeitlich den frühen Beispielen um 1500 folgt ein Fries in der Spiegelstube des Großen Wendelsteins am Schloss Hartenfels aus dem Jahre 1537. Er kann aufgrund von schriftlichen Quellen der Cranach-Werkstatt zugeschrieben werden. Die Gesellen Lucas Cranachs d. Ä., Paul Ryß und Hans Rentz, sowie der Lehrknabe Bartel waren drei Wochen mit der Vergoldung des Gesims und der Ausführung des Frieses beschäftigt. Lucas Cranach selbst war vier Tage anwesend, als er die gemalten acht Tücher für die Decke nach Torgau gebracht hat. ${ }^{17}$ Erhalten ist nur der Groteskenfries, der in Grauschwarz angelegt ist und darüber mit gebundenem Azurit und Ölvergoldung auf Massicot vervollkommnet wurde. Unter der Balkendecke zieht sich ein illusionistisch gemaltes Gesims entlang.
Torgau, Haus Pfarrstraße 3, erstes Obergeschoss, Große Stube, Wandgemälde mit einer auf einer Steinbrüstung stehenden Wappenhalterin, um 1500 Foto: Angelica Dülberg

5 Christoph Gasser/Helmut Stampfer: Die Jagd in der Kunst Alttirols. Bozen 1994, Abb. $134 \mathrm{f}$.

6 Gasser/Stampfer 1994 (wie Anm. 5), Abb. 142 f., 144 f.

7 Gasser/Stampfer 1994 (wie Anm. 5), Abb. $152 \mathrm{f}$.

8 Gasser/Stampfer 1995 (wie Anm. 5), Abb. $154 \mathrm{f}$.

9 Erika Kustatscher/Angela Möller/Roland Möller/Helmut Stampfer: Der Jöchlsthurn in Sterzing. Innsbruck/ Wien/Bozen 1992, S. 114123.

10 Eva Börsch-Supan: Garten-, Landschafts- und Paradiesmotive im Innenraum. Eine ikonographische Untersuchung. Berlin 1967, S. 233239.

11 Dülberg 2004 (wie Anm. 4), S. $123-125$, Abb. S. 122; vgl. auch Mechthild Noll-Minor: Das Freihaus Pfarrstraße 3. In: Torgau - Stadt der Renaissance. 2. Auflage Dresden 2004, S. 146-153, Abb. 5-8.

12 Vgl. Maximilian Gritzner: Geschichte des sächsischen Wappens. Sonderdruck aus der Vierteljahresschrift für Wappen-, Familien- und Siegelkunde. Berlin 1900/01; Dülberg 2004 (wie Anm. 4), S. 124.

13 Vgl. Angelica Dülberg: Zur Ikonographie der Wandmalereien im zweiten Obergeschoss. In: Kuratorium „Rettet Meißen - Jetzt“ (Hrsg.) Meißen Rote Stufen 3. Restaurierung einer spätgotischen Bohlenstube. Meißen 2016, S. 8-11; Angelica Dülberg: Die sogenannte Schatzkammer im Kaufmannshaus Untermarkt 5 in Görlitz. Zur Ikonographie der illusionistischen Wandmalereien vom Anfang des 16. Jahrhunderts. In: Marburger Jahrbuch für Kunstwissenschaft 28 (2001), S.133-158

14 Dülberg 2001 (wie Anm. 13), S. 151, Anm. 88 f.; Eva Steinberger: Eine malerische Raumausstattung im Regensburger Bürgerhaus Bachstraße 8. In: Jahrbuch der Bayerischen Denkmalpflege 39 (1988), S. 73-92.

15 Vgl. Noll-Minor 2004 (wie Anm. 11), S. 147, 150. 


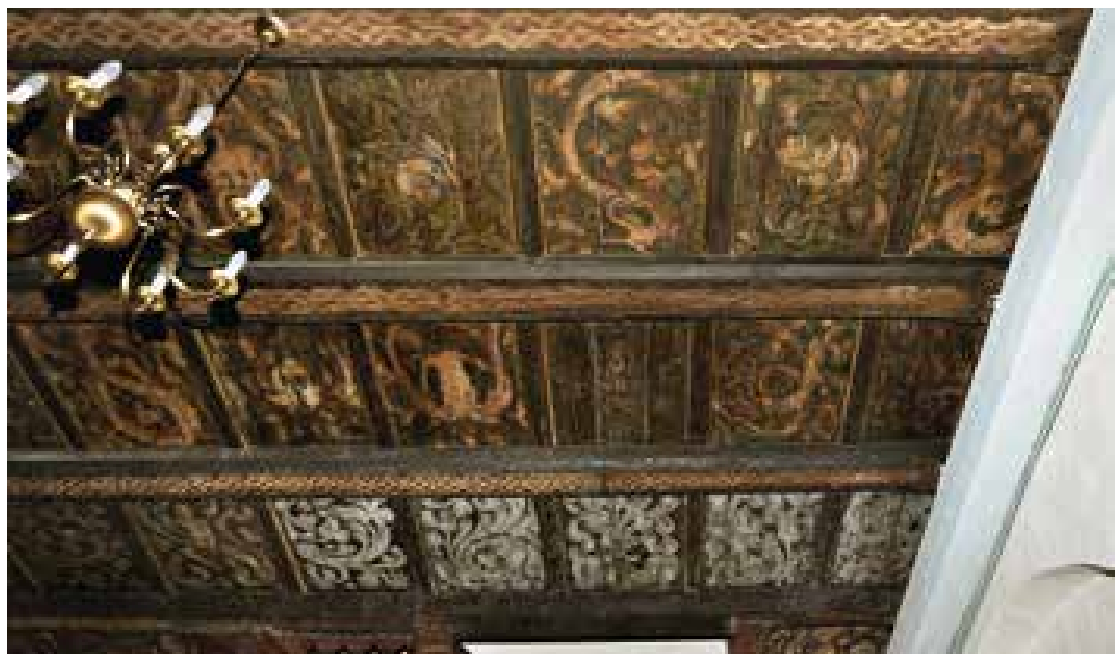

Torgau, Breite Straße 9, Bürgermeister-Ringenhain-Haus, Treppenhausdiele des ersten Obergeschosses, Holzbalkendecke, Arabeskenmalerei mit eingewobenen Groteskenmotiven, nach 1596

Foto: Claudia Herrmann

16 Fritz Bellmann/Marie-Luise Harksen/ Roland Werner: Die Denkmale der Lutherstadt Wittenberg. Weimar 1979, S. 84, Abb. 65 f.

17 Vgl. Mechthild Noll-Minor: Die Spiegelstube im Großen Wendelstein des Schlosses Hartenfels zu Torgau. In: Sächsische Heimatblätter 42 (1996), Heft 4, S. 209-217.

18 Vgl. Thomas Schmidt: Die historischen Fassungsbefunde in den Häusern IA und IB. In: Cranach-Stiftung (Hrsg.): Lucas Cranach d. ̈̈. und die Cranachhöfe in Wittenberg. Halle 1998, S. 119-122, Abb. 98-101.

19 Vgl. Beitrag von Nadja Kühne in diesem Heft.

20 Angelica Dülberg: Das Torgauer Renaissancehaus Breite Straße 9. Ein „Kleinod“ unter den Bürgerhäusern der Renaissance. In: Denkmalpflege in Sachsen 2002. Mitteilungen des Landesamtes für Denkmalpflege Sachsen. Beucha 2003, S. 34-48, hier S. 37 f., Abb. 8 f.; Dülberg 2004 (wie Anm. 4), S. 126, Abb. 4 f.; Dülberg 2015 (wie Anm. 4), S. 210-212, Abb. 4.

Torgau, Breite Straße 9, Bürgermeister-Ringenhain-Haus, Treppenhausdiele des zweiten Obergeschosses, Ausschnitt der unter einer Unterhangdecke wieder zutage getretenen Deckentafeln, nach 1596 Foto: Andreas Dubslaff
Darunter befindet sich ein Groteskenfries mit sich wiederholenden, jedoch stets variierten Volutenspangen, aus denen sich Blattranken, trötenförmige und pokalartige Füllhörner, aus denen wiederum Blattlaub sprießt, sowie in Blattranken auslaufende Tier-und Menschenköpfe entwickeln. Dazwischen schweben im Profil gegebene Cherubköpfe. Im ersten Obergeschoss des Cranachhofes Markt 4 in Wittenberg ist eine Deckenbemalung aus etwa derselben Zeit erhalten, die im Prinzip ähnlich gestaltet ist. ${ }^{18}$ In den ehemals tiefblauen Feldern zwischen einem in Grau gemalten Rippengewölbe erscheinen Varianten eines sich stets wiederholenden, offensichtlich hellen Stein imitierenden Groteskenmotivs, deren Köpfe vogelähnlich gestaltet sind und in arabeskenähnlichen Voluten auslaufen, an deren Enden ockerfarbene Dreiblätter sitzen. Auf daneben liegenden Feldern rankt ockerfarbenes Weinlaub. In der Spiegelstube sind auf dem grauschwarzen Hintergrund Reste des blauen Azurits erhalten, auch sind schwarze Schlagschatten noch ablesbar. Sie weisen wie auch an der Wittenberger Decke auf eine beabsichtigte illusionistische Wirkung als Relief zwischen den gemalten Rippen oder hier unter dem gemalten Gesims hin. Im Jahr 2017 wurde zudem eine prunkvolle, illusionistisch gemalte Bekrönung des kleinen spitzbogigen Eingangsportals in die Spiegelstube freigelegt und der gesamte Raum restauratorisch instandgesetzt. ${ }^{19}$

Eine phantasievolle Steigerung der in arabeskes, üppiges und dickblättriges Rankenwerk eingewobenen grotesken Motive - verschiedenartige Masken, Drolerien, Fabelwesen sowie menschliche Körper, deren Gliedmaßen vegetabil weiterwachsen - befindet sich an der Decke der großen Treppenhausdiele im ersten Obergeschoss des Torgauer Bürgermeister-Ringenhain-Hauses Breite Straße $9 .{ }^{20}$

Es handelt sich hier allgemein um eine Stilrichtung der in Arabesken eingewobenen Grotesken, die insbesondere die sogenannten Kleinmeister in ihren kleinformatigen Ornamentdrucken um $1530 / 40$ geprägt haben. ${ }^{21} \mathrm{Zu}$ ihnen gehörte unter anderen neben Barthel und Sebald Beham auch Heinrich Aldegrever. Akanthus-Wellenranken werden mit anderen Motiven, wie vor allem vegetabilisierte Wesen und solche mit Schlangenleibendungen, die wellenrankengleiche Bewegungen vollziehen, kombiniert. Weil sich derartige Motive über mehrere Jahrzehnte gehalten haben, kann man die Deckenmalereien durchaus in unmittelbare Zeitnähe - um 1600 - zu denen in der Treppenhausdiele des zweiten Obergeschosses rücken.

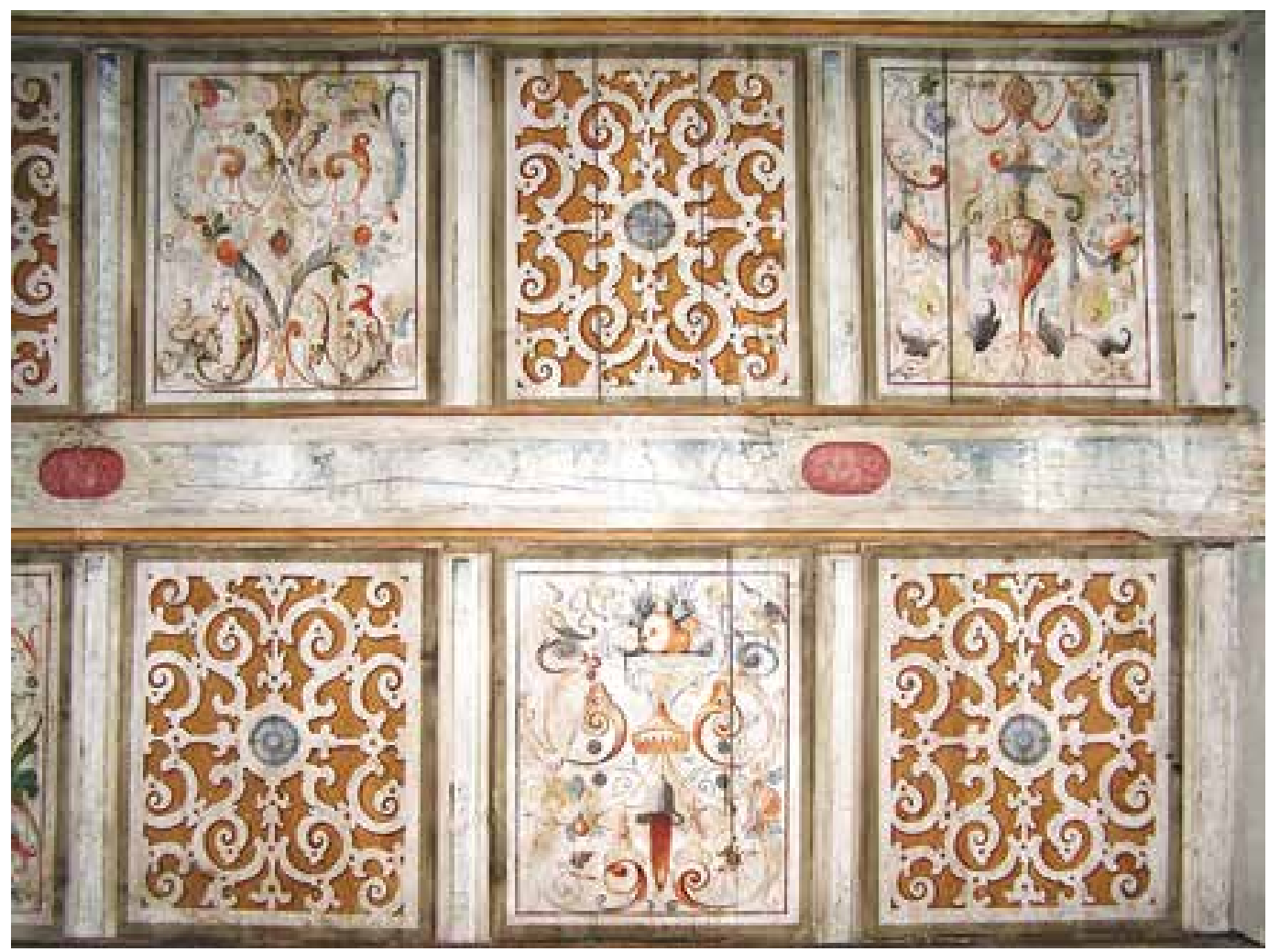


Diese unterscheidet sich in vielerlei Hinsicht, gleichwohl sticht sie aus allen mir bekannten bemalten Holzbalkendecken in Bürgerhäusern aus der zweiten Hälfte des 16. oder dem Anfang des 17. Jahrhunderts heraus, vor allem aufgrund ihrer hervorragenden malerischen Qualität. ${ }^{22}$ Obwohl auch hier wie im ersten Obergeschoss aufgrund einer Behandlung mit ölhaltigen Substanzen in den zwanziger oder dreißiger Jahren des zwanzigsten Jahrhunderts alle damals sichtbar gewesenen Tafeln heute eher als Braun-in-Braun-Malerei erscheinen, vor allem das Grün in Schwarz umgeschlagen ist, das Blau und das Rot verblasst oder ganz vergangen sind, haben die Tafelbilder trotzdem ihren besonderen Reiz der variationsreichen Groteskenmalerei nicht verloren. Vor einigen Jahren hat Peter Ehrhardt ebenso wie im ersten Obergeschoss das Erscheinungsbild restauratorisch wesentlich verbessert.

Nachdem er in einem später durch eine Wand abgetrennten Teil der Treppenhausdiele die im Barock eingezogene Decke entfernt hatte, kamen weitgehend im Originalzustand erhaltene Deckentafeln zum Vorschein. Hier werden die ursprüngliche Farbigkeit, die Motivvielfalt sowie die Virtuosität der fast frei aus der Hand gemalten Groteskendarstellungen erst erlebbar. Die nun wieder zutage getretenen Tafeln zeigen auf weißem Grund insbesondere zart abgestufte Blau-, Grün-, Rot-, Braun- und Gelbtöne, denen mit feinen Schraffuren Binnenzeichnungen und Lichter aufgesetzt sind, die eine außergewöhnliche Spontaneität und Lebendigkeit hervorrufen sowie das Roll-, Schweifund Beschlagwerk plastisch erscheinen lassen. Bis auf wenige Zirkelstriche mit einem silberstiftähnlichen Material oder sehr fein angespitztem Rötelstift bei den mit ihnen wechselnden rein ornamentalen Tafeln ist keine Unterzeichnung zu erkennen. Viele einzelne Motive sind äußerst versiert mit einer enormen Feinheit gemalt, die an einer Decke erstaunlich ist. Betrachtet man zum Beispiel den in

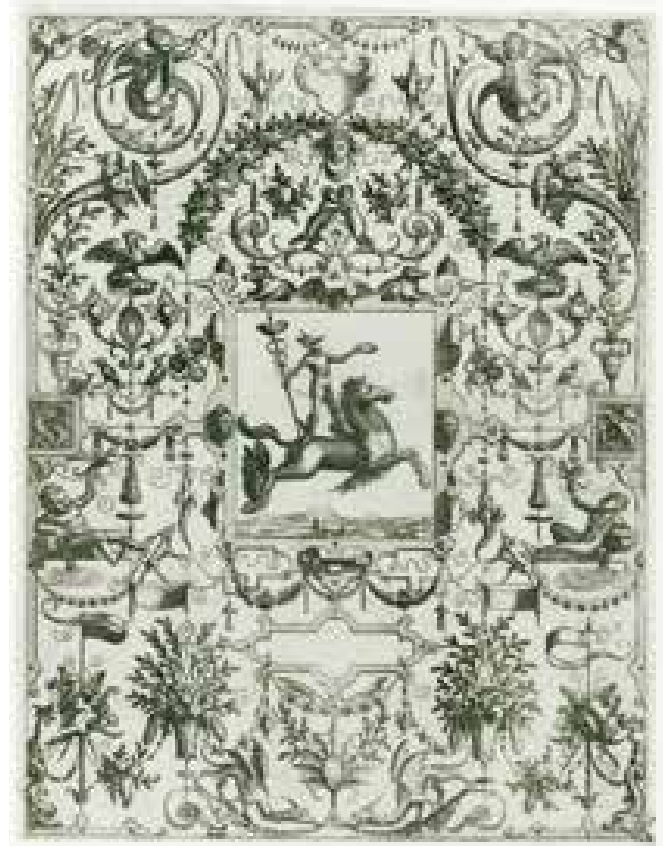

einer Glocke hängenden weichen Fuchsschwanz oder auf einer Tafel im vorderen Teil der Diele das feine Fell eines springenden Steinbocks, der gleichzeitig eine hervorragende Tierdarstellung als solche ist. Wirkliche Tiere, die häufiger im vorderen Teil der Diele auftreten, aber auch Fabeltiere sind ebenso gekonnt gemalt. Der Künstler beherrschte zudem alle Facetten der Groteskenmalerei mit leichter Hand und perfekt, wenn man sich zum Beispiel den aus einer Volute hervorwachsenden Fratzenkopf ansieht. Er war fähig, souverän mit einem reichen Formenschatz zu spielen.

Wenn auch die groteske Malerei wie üblich aus einer vertikalen Mittelachse als Grundgerüst aufgebaut ist, so entspricht sie hier nicht der oft gängigen strengen Symmetrie, vielmehr sind - auch mit malerischen Mitteln - bewusst Asymmetrien hervorgerufen worden. Stoffbänder, Voluten wie auch vegetabile und figürliche Elemente sind links und rechts der Mittelachse stets differenziert gestaltet, was zusätzlich die erwähnte Lebendigkeit der Malerei unterstreicht.

Ursprünglich dachte man an einen italienischen Maler, zumindest an einen „unverkennbar italienischen Einfluß ${ }^{\prime 2} .{ }^{23}$ Schließlich fand ich die unmittelbaren Vorbilder, die sogenannte Grotteschi-Serie „Diversche Grotesken“ von Hans Vredeman de Vries, die zwischen 1565 und 1570 erschienen ist und neben dem Titelblatt 16 Blatt umfasst. ${ }^{24}$

Hervorgehoben sei zunächst, dass der Maler - wie es meistens üblich ist - keineswegs das gesamte Graphikblatt kopierte, sondern lediglich Hauptmotive im Mittelpunkt der Grotesken, wie den Kentaurenkampf oder die Frau auf dem Kamel, aber auch kleine, in die Gesamtgestaltung der eigentlichen Groteske eingebundene Motive, wie Fabeltiere und Phantasievögel, den Adler auf dem Baldachin oder groteske Fratzen, übernommen hat und diese in ein frei erfundenes neues System seiner Groteskenmalerei integrierte. Mit malerischen Mitteln ging er dabei weit über die graphischen

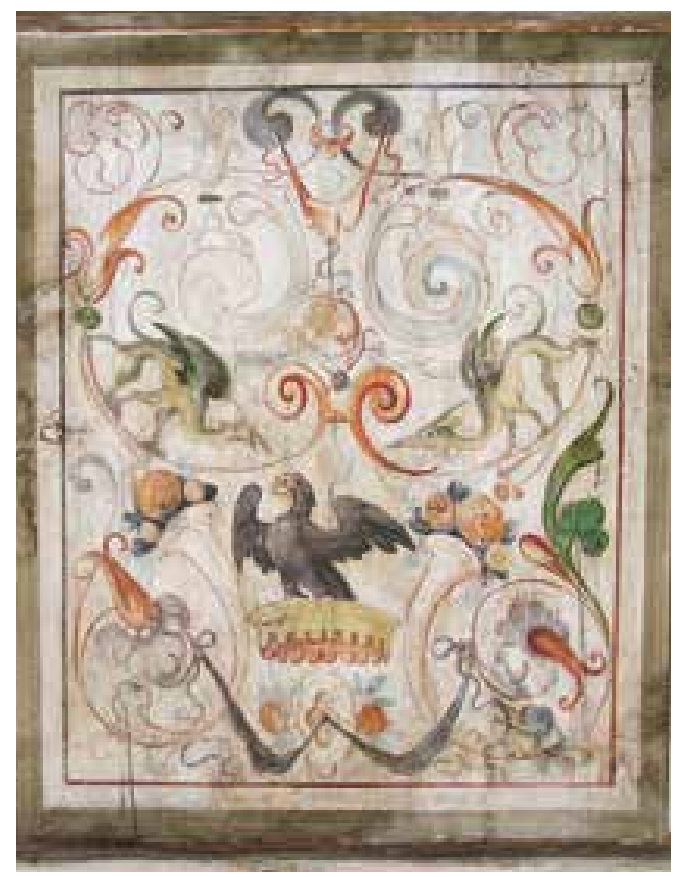

21 Vgl. u. a. den Artikel „Kleinmeister“. In: Lexikon der Kunst. Bd. 3. Leipzig 1991 S. $177 \mathrm{f}$.

22 Dülberg 2002 (wie Anm. 20), S. 38-47, Abb. 10-30; Dülberg 2004 (wie Anm. 4) S. 127-132, Abb. 7-17; Dülberg 2015 (wie Anm. 4), S. 212-218, Abb. 5-11.

23 W. Wollschläger: Ueber die Kassettenmalerei im Haus Breitestraße Nr. 354 [heute Nr. 9]. In: Veröffentlichungen des Altertumsvereins der Stadt Torgau 5 (1893), S. 15-22, hier S. 18.

$24 \mathrm{Ihr}$ genauer Titel lautet: "Grottesco in Diversche Manieren“. Vgl. Carsten-Peter Warnke: Die ornamentale Groteske in Deutschland 1500-1650. Bd. 1. Berlin 1979, S. 32, Abb. 225-232; Peter Fuhring: Hollstein's Dutch and Flemish etchings, engravings and woodcuts, 1440-1700. Bd. 47: Hans Vredeman de Vries. Rotterdam 1997, S. 215231, Kat. Nrn. 240-256 Hans Vredeman de Vries und die Renaissance im Norden. Ausstellungskatalog des Weserrenaissance-Museums Schloss Brake, Lemgo. München 2002, S. 248-251, hier Kat.-Nr. 82.

links: Hans Vredeman de Vries, "Grottesco in Diversche Manieren", Blatt 17, Antwerpen, um 1565/70, Amsterdam, Rijksmuseum-Stichting - Amsterdam, RijksmuseumStichting

Torgau, Breite Straße 9, Bürgermeister-Ringenhain-Haus, Treppenhausdiele des zweiten Obergeschosses, Deckengemälde mit zwei Fabeltieren und einem Adler, nach 1596 Foto: Peter Ehrhardt 
25 Freundlicher Hinweis von Jürgen Herzog, April 2002.

26 Sächsisches Staatsarchiv, Hauptstaatsarchiv Dresden (HStA Dresden), 10024 Geheimer Rat (Geheimes Archiv), Loc. 35623, Nr. 16, f. 62 .

27 Cornelius Gurlitt: Beschreibende Darstellung der älteren Bau- und Kunstdenkmäler des Königreichs Sachsen. Bde. 21-23. Stadt Dresden. Dresden 1903, S. 416.

28 Karl Berling: Die Dresdner Malerinnung. In: Neues Archiv für Sächsische Geschichte und Altertumskunde 11 (1880), S. 263-281, hier S. 273, Anm. 14

29 Was offensichtlich nicht stimmt. Vgl. HStA Dresden, 10024 Geheimer Rat (Geheimes Archiv), Loc. 1872/3, f. 6, Nr. 12 b.

30 Vgl. Ernst Sigismund: Bruck (Brugk), Peter de. In: Thieme-Becker. Allgemeines Künstlerlexikon. Bd. 5. Leipzig 1911, S. 94; Frauke Hinneburg: Bruck (Brug, Brück), Peter (Pieter) de. In: Saur. Allgemeines Künstlerlexikon. Bd. 14. München/ Berlin 1996, S. 462. Roland Werner: Das Lutherhaus in Wittenberg. Leipzig 1971 (ohne Seitenangaben), schrieb die Bemalung der Decke und der Wände in der Lutherstube Pieter de Bruck zu, weil er eine ,auffallende Ähnlichkeit“ mit den Deckenmalereien im Torgauer Haus Breite Straße 9 sah. Vgl. auch Bellmann/Harksen/Werner 1979 (wie Anm. 16), S. 44 f. Ich kann die enge Verwandtschaft der beiden Deckenbemalungen nicht erkennen.

31 Sein Aufsatz „Die Decke der Engelstube und der Maler Nikolaus Rosman“ wird in der Monographie „Das Bürgermeister-Ringenhain-Haus in Torgau" erscheinen.

32 Peter Findeisen/Heinrich Magirius: Die Denkmale der Stadt Torgau. Leipzig 1976, S. 185-187.

Torgau, Breite Straße 9, Bürgermeister-Ringenhain-Haus, erstes Obergeschoss, nördlicher Seitenflügel, sogenannte "Trinkstube", Jagdfries, um 1620 Foto: Claudia Herrmann
Vorbilder hinaus. Zu bedenken ist auch, dass der Maler verhältnismäßig kleine, ja winzige Vorbilder in seine weitaus größeren Gemälde umsetzen musste, was ihm meisterhaft gelungen ist, wie man im Vergleich sehen kann.

Während in der Grotteschi-Serie die Hauptszenen durch Beschlagwerk oder architektonische Umrahmungen herausgehoben sind, werden sie an der Decke frei in die umgebenden Grotesken integriert. Häufig stehen sie auf stets anders verzierten kleinen Podesten, Schalen oder einfach auf dem Rollwerk. Der Maler lässt durchaus auch Details seiner Vorbilder fort. Der Rückenfigur eines Kriegers, der sich auf seinen Schild stützt, fehlt nicht nur die Stele, sondern auch die Standarte. Außerdem ist er nicht nackt wie im Stich, sondern trägt ein kurzes gelbes Hemdchen. Möglicherweise ist hier der Helm bewusst verändert: Die rechte Kinnwange ist zum Spitzbart des hier im Profil gegebenen Gesichts geworden, an den der Krieger mit seiner Rechten greift, während er im Stich einen Zweig hält.

Blatt 17 weist gleich mehrere Motive auf, die in verschiedene Gemälde übernommen worden sind. Von dem Mittelbild eines auf einem springenden Pferd stehenden Merkur verwendete der Maler lediglich das Pferd, dessen Hinterbeine hier spitz in eine große röhrenartige Volute hineinstechen. Der am linken Rand auf einem Beschlagwerksteg lagernde Putto, der in der Rechten ein Gefäß hält, aus dem Wasser fließt und auf dessen linker Hand eine Libelle sitzt, ist im Gemälde zum mittleren Hauptmotiv geworden und räkelt sich hier auf einer Muschelschale, in die das Wasser fließt. Auch für den schwarzen Adler, der flatternd auf einem Baldachin Platz genommen hat, und die beiden über ihm befindlichen geflügelten Fabeltiere - im Stich am unteren Rand - diente die Graphik als Vorlage. Hier wird wieder deutlich, wie der Künstler sie mit malerischen Mitteln geradezu zum Leben erweckt hat.

Die auf Blatt 2 der Serie lediglich als kleine Randszene kaum auffallenden Grotesken zweier in einer Rollwerkkartusche einen Handstand machenden Gnome mit Schlangenleibern und die im Profil gezeigten Fratzen sind an der Deckentafel zum Hauptmotiv des unteren Bereiches geworden und wirken somit wesentlich prägnanter. Ja, der Maler steigert das Groteskenhafte noch, indem er der rechten Fratze ein rotes Zipfelmützchen aufsetzt. In ähnlicher Weise wählte er ein kleines Randmotiv von Blatt 13 zur Hauptdarstellung einer anderen Tafel aus, auf der ein Satyr einem auf ihn zu springenden Steinbock einen Schild entgegenhält. Auf die hervorragende Wiedergabe des Tieres habe ich bereits hingewiesen.

Es ließen sich noch weitere Vergleichsbeispiele finden, vor allem einzelne Tierdarstellungen, die in gewisser Weise anregend für die zentralen Motive der Malereien gewesen sein mögen, die der Künstler jedoch aus eigener Kenntnis wesentlich lebensnaher gestaltete. So befinden sich zum Beispiel zwei verschiedenartige Hühnervögel als zentrales und äußerst großes Mittelmotiv auf den Deckentafeln. Vögel waren offensichtlich sehr beliebt, so erscheinen auch zwei Papageien und ein Käuzchen. Ebenfalls kommen häufiger Affen vor. Alle diese Darstellungen weisen den Künstler der Decke als guten Tiermaler aus, der auch die arttypischen Details und Verhaltensweisen genau kannte. Diese Feststellung ist durchaus von Bedeutung bei der Suche nach dem Schöpfer der Malereien.

Einen mit Namen bekannten Maler als Schöpfer ausfindig zu machen, ist aufgrund der schlechten Quellenlage überaus schwierig. Doch erfahren wir, dass ein Maler mit Namen Pieter (Peter) de Bruck (Brück, Brugk) am 15. Dezember 1594 seine Tochter Catarina in Torgau taufen ließ. Er war also zur gegebenen Zeit in der Stadt. ${ }^{25}$ Vermutlich handelt es sich um einen Bruder des Torgauer Bildhauers Ägidius de Bruck, der aus Lüttich stammte. Pieter de Bruck wird dann zum ersten Mal im Zusammenhang der Renovierung des Dresdner Residenzschlosses und des Kanzleihauses im Jahre 1602 genannt. ${ }^{26}$ Er gehörte zu einer Gruppe auswärtiger Maler und wird als Torgauer bezeichnet. Zusammen mit Christoff Gromm malte er 1609/10 das „Fürstliche Haus auf der Elbgasse“ (Schlossstraße 30) aus. ${ }^{27}$ Er wurde 1619 Bürger von Dresden und 1620 Mitglied der Dresdner Malerinnung. ${ }^{28}$ Am kursächsischen Hof war er als Tiermaler tätig und lieferte zwischen 1620 und 1623 entsprechende Werke, von denen wir heute leider keine Kenntnis haben. 1626 soll er Malereien an der Orgel der Sophienkirche ausgeführt haben. ${ }^{29}$ 1627 war er wieder in Torgau, wurde dort Bürger

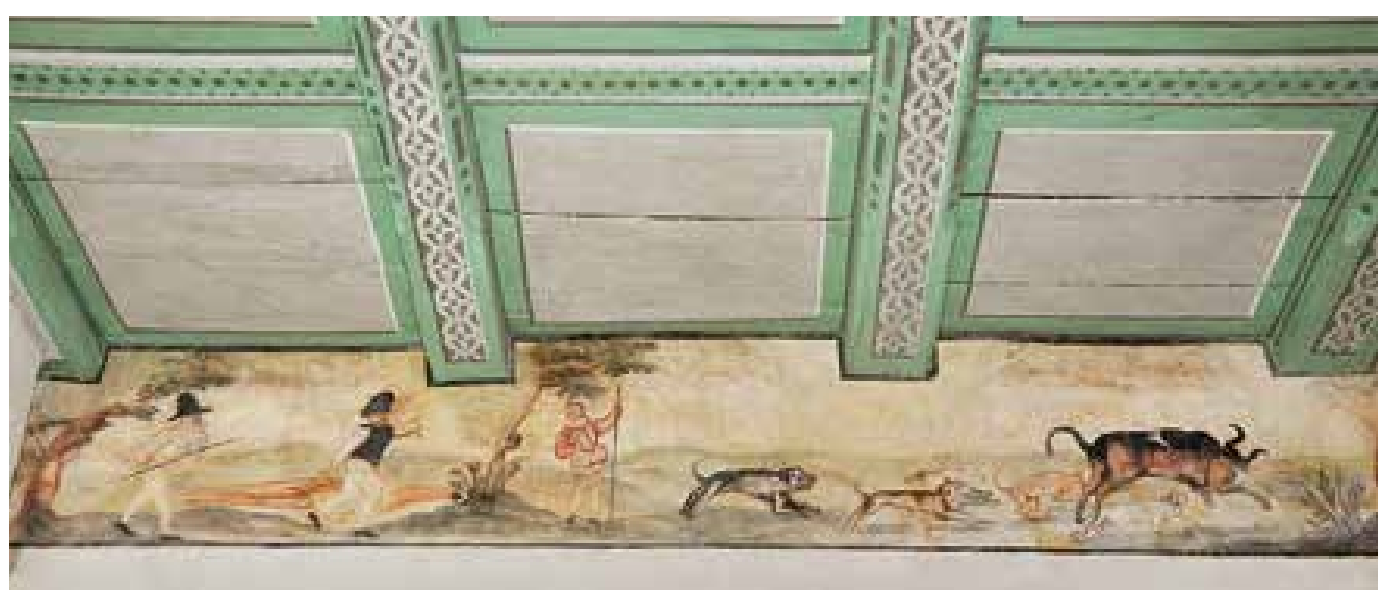




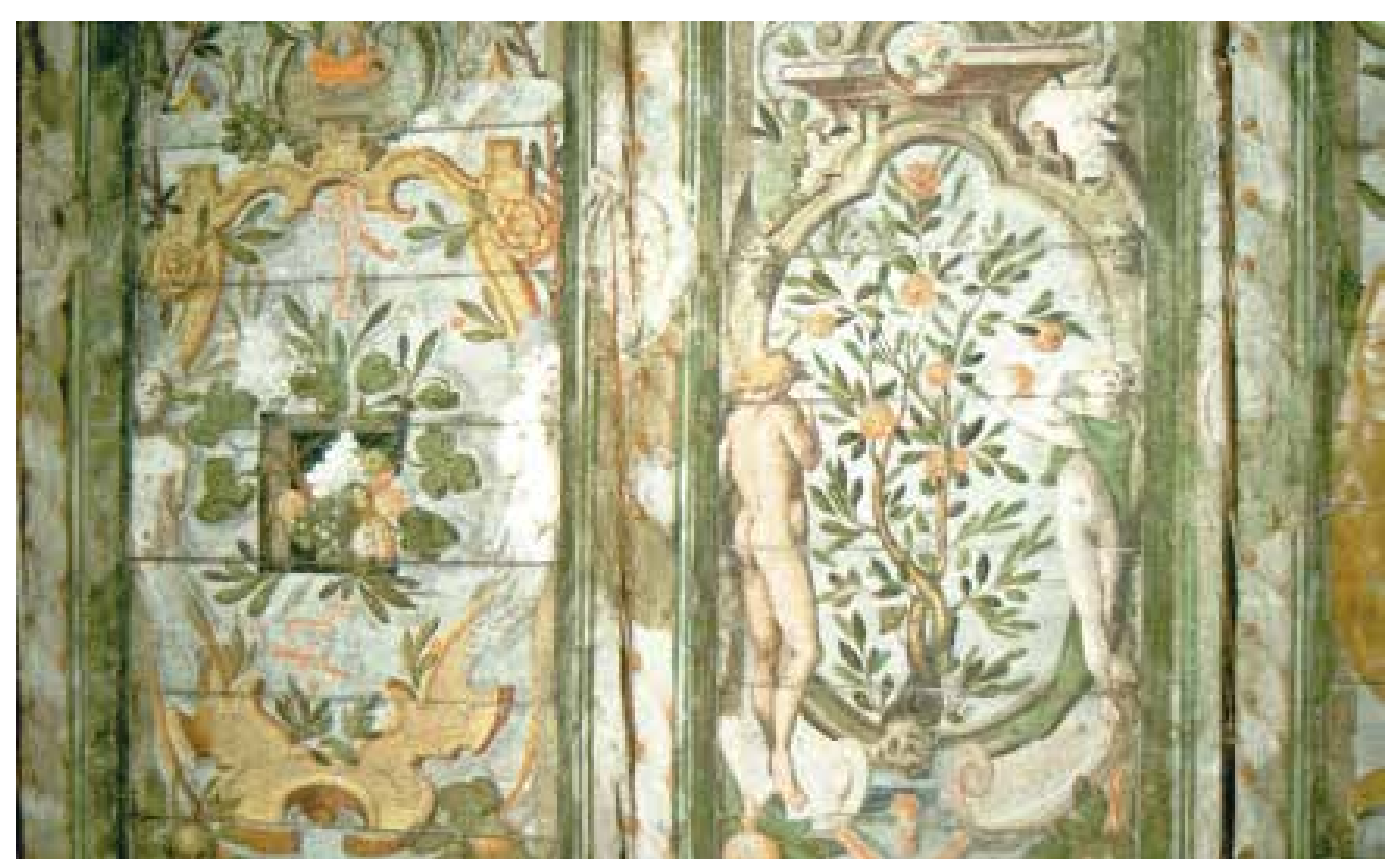

und Malerältester. ${ }^{30}$ Mehr ist über Pieter de Bruck bis jetzt nicht bekannt.

Von Bedeutung scheint mir, dass er vor allem als Tiermaler ausgewiesen ist, konnten wir doch feststellen, dass der Schöpfer der Deckenmalereien eine besondere Fähigkeit für dieses Genre zeigt. Obwohl Pieter de Bruck bisher kaum bekannt ist, war er ein versierter Maler, der immerhin mit der Ausmalung des Fürstlichen Hauses beauftragt wurde und auch für den Dresdner Hof Werke schuf.

Im Hauptraum des ersten Obergeschosses sticht eine Holzbalkendecke mit 44 bemalten Kassettentafeln hervor, in denen jeweils geflügelte Putten in grauweißen Wolkengebilden vor einem dunkelblauen Himmel mit goldenen Sternchen schweben. Sie halten entweder Leidenswerkzeuge wie den Essigschwamm, die Martersäule oder die drei Nägel sowie auch verschiedenartige Musikinstrumente. Sebastian Schulze hat die offensichtlich 1631 entstandene Decke der Engelstube ausführlich untersucht und den Hallenser Maler Nikolaus Rosman mit Gehilfen als ihren Schöpfer erkannt. ${ }^{31}$ Im Schloss Hartenfels sind quellenmäßig vier Räume überliefert, die mit „gewölke und Engelein gemahlet" und möglicherweise vorbildhaft waren. ${ }^{32}$

In allen Räumen vom Erdgeschoss bis zum zweiten Obergeschoss des Bürgermeister-RingenhainHauses wurden im Laufe der Jahre seit 1995 unter eingezogenen Unterhangdecken und Übermalungen Deckenmalereien aus der Zeit um 1600 wiederentdeckt. Die hier nicht besprochenen zeigen ausschließlich ornamentale Malereien wie Ranken und Schweifwerk, die im Vergleich mit anderen Beispielen in Torgau und Sachsen in ihrer Qualität ebenfalls herausragen. Auch Teile der ehemaligen Wandmalereien wurden unter entfernten Tünchen wieder freigelegt. Hier sei lediglich auf diejenigen in der sogenannten Trinkstube im ersten Obergeschoss des nördlichen Seitenflü- gels hingewiesen, weil sie für den bürgerlichen Bereich durchaus außergewöhnlich sind. An der Süd- und Nordwand stehen lebensgroße Wächterfiguren - ein Hellebardier und ein Musketier. Ersterer hält eine Helmbarte in der Rechten, der andere ist mit einer Muskete und einer Musketengabel ausgestattet. ${ }^{33}$ Aus der Zeit um 1600 sind ähnliche Figuren, sogenannte Türsteher, vor allem im Schloss Schmalkalden, im Venussaal des Schlosses Augustusburg und im Schloss Elbogen (Loket) in Böhmen bekannt. Auch der wandabschließende Jagdfries ist vor allem in Schlossbauten zu finden, so in den Schlössern Nossen und Rochsburg, aber auch in zwei Freiberger Bürgerhäusern - Obermarkt 8 und Pfarrgasse $11 .^{34} \mathrm{Ne}$ ben Rollwerk mit Zweigen und Singvögeln in der Fensterlaibung sind an der Nord- und Südseite noch Muschelnischen in einer hölzernen Scheinwerk und eine Tierdarstellung (Bär?) hinter Gitterstäben einer Nische erwähnenswert. täfelung sowie im Sockel Löwenköpfe im Ranken-
Torgau, Haus Scheffelstraße 1 . erstes Dachgeschoss, Deckengemälde mit Beschlagwerkkartuschen und Früchtebündeln, 1620/30 Foto: Angelica Dülberg

33 Vgl. Yvonne Fritz: Torgauer Stadttrabanten bei der Hochzeit von Herzog Johann Georg 1607. In: Churfürstliche Guardie. Die sächsischen Kurfürsten und ihre Leibgarden im Zeitalter der Reformation. Dresden 2012, S, 56-59, Abb. 30.

34 Dülberg 2004 (wie Anm. 1), S. 850 f., Abb. 1353-1362.

35 Marjolein Leesberg: Karel van Mander (The new Hollstein Dutch \& Flemish etchings, engravings and woodcuts 1450-1700). Rotterdam 1999, S. 176-178, Abb. 152 155; Jan Pieter Filedt Kok/ Marjolein Leesberg: The Gheyn Family. Part I (The new Hollstein Dutch \& Flemish etchings, engravings and woodcuts 1450-1700). Rotterdam 2000, S. 194-199, Abb. 127-130.

36 Vgl. Dülberg 2004 (wie Anm. $4)$.

Torgau, Rosa-Luxemburg-Platz 4, Handwerkerhaus Am Bäckerwall, an die Beillinien des Fachwerks angehängte Blüten, um 1620 Foto: Angelica Dülberg

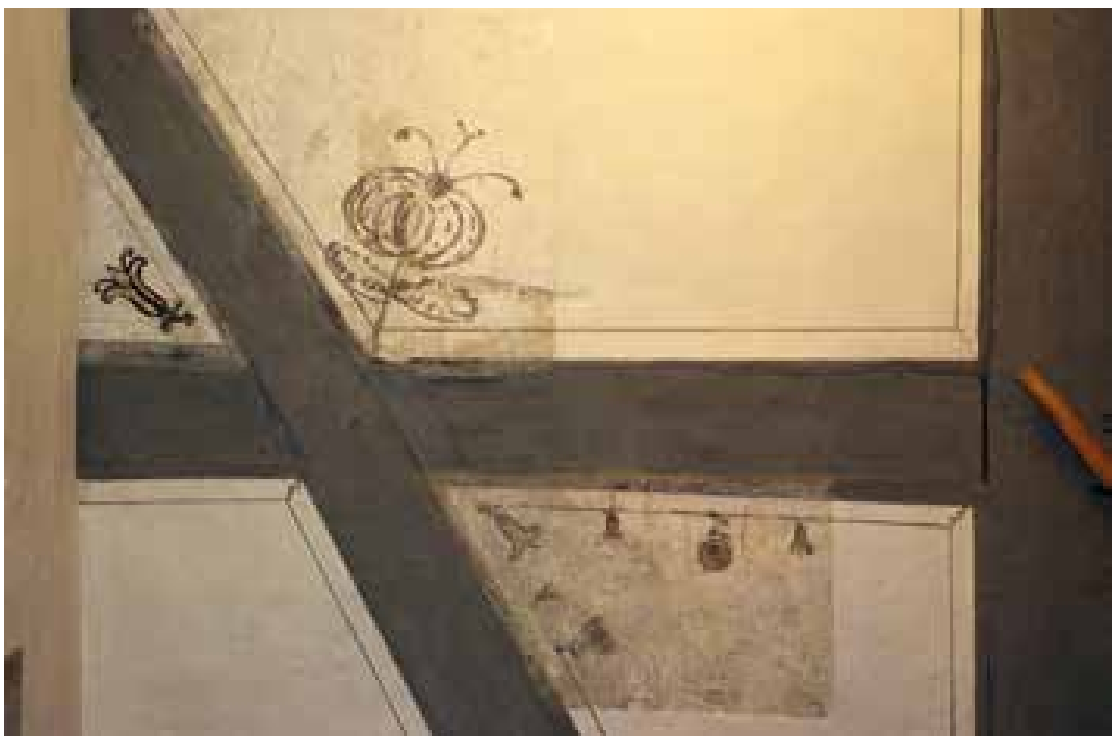




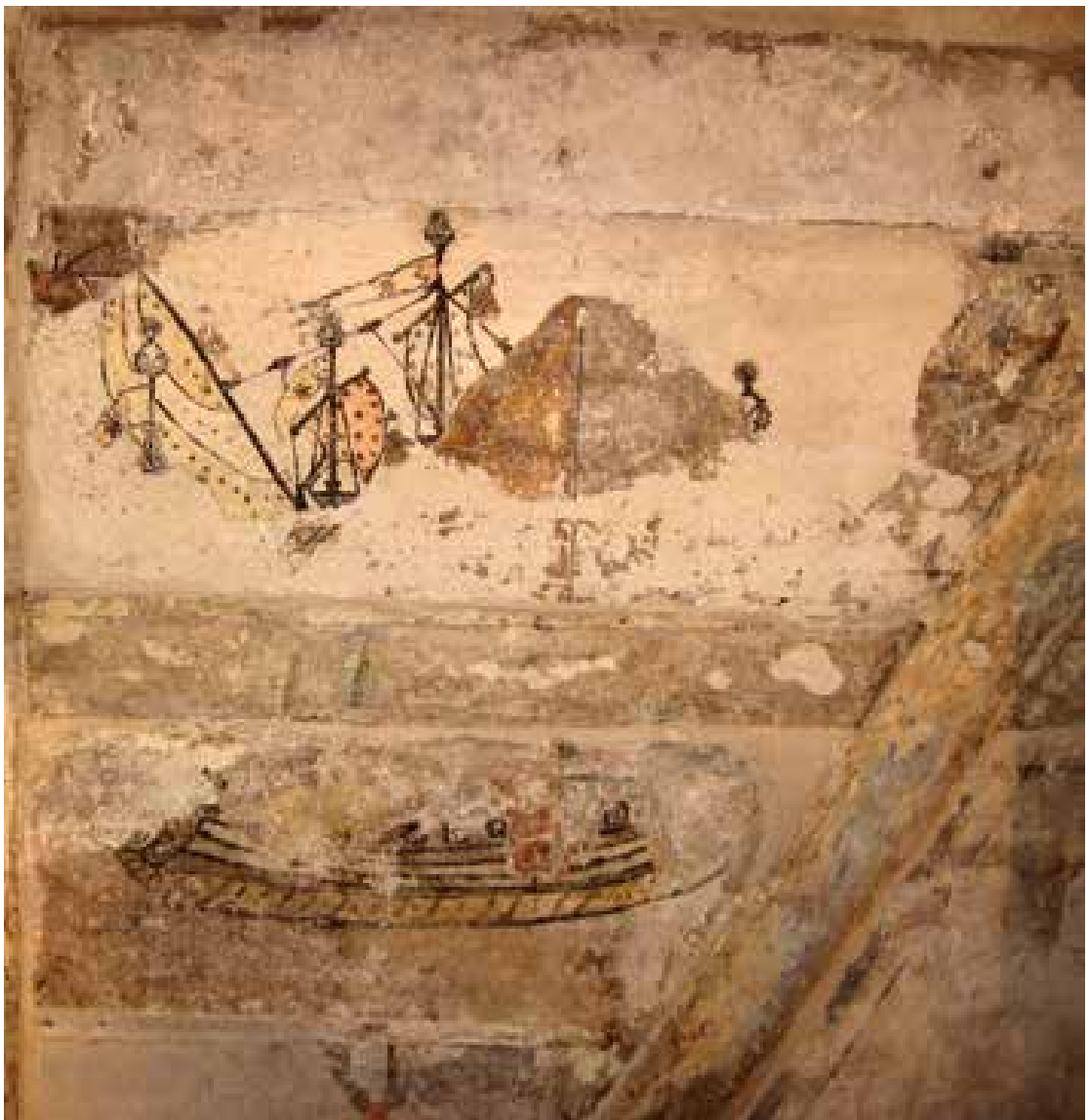

Torgau, Rosa-Luxemburg-Platz 4, Handwerkerhaus Am Bäckerwall, dreimastiges Segelschiff, um 1620

Foto: Angelica Dülberg

37 Jürgen Herzog: Das Handwerkerhaus „Am Bäckerwall“ in Torgau. In: Sächsische Heimatblätter 56 (2010), Heft 4, S. 310-328; Stadtverwaltung Torgau (Hrsg.): Haus „Am Bäckerwall“. Handwerkerhaus des 17. Jahrhunderts. Torgau 2010.

38 Albrecht Sturm: Das Haus Markt 14 und die Geschichte seiner Holzbalkendecken. In: Pirnaer Hefte 6 (2006), S. 51-68, hier S. 59-62, Abb. 73-83, hier Abb. 75.

Autorin

Prof. Dr. Angelica Dülberg Dresden
In Sachsen haben sich durchaus einige bemalte Türen aus der Renaissance erhalten, allerdings fast ausschließlich mit ornamentalen Motiven, wie zum Beispiel im Schloss Augustusburg mit Mauresken und im Haus Breite Straße 9 mit Arabesken und Schweifwerk. Deshalb stellen zwei Türen im ersten Obergeschoss eine Besonderheit dar. Nach der Freilegung von Übermalungen zeigten sich drei vollwertige Gemälde mit drei von vier Darstellungen der Elemente Luft, Feuer und Erde. Vom vierten Element Wasser fanden sich keine Spuren, man ergänzte es mit einer Vergrößerung des vorbildhaften Kupferstichs. Die Türgemälde gehen auf von Jacques de Gheyn Il nach den Inventionen Karel van Manders um 1590 geschaffenen Kupferstichen zurück $^{35}$ und orientieren sich mit großer Treue an den Vorbildern.

Neben den beiden hervorragenden Häusern Breite Straße 9 und Pfarrstraße 3 gibt es in Torgau noch weitere Bürgerhäuser, die Wand-und Deckenmalereien aus dem Ende des 16. oder dem Anfang des 17. Jahrhunderts aufweisen. ${ }^{36}$ Varianten reicher Maureskenmalereien an den Holzbalkendecken finden wir in den Häusern Breite Straße 22, Nonnengasse 11 und Pfarrgasse 3. Offensichtlich war hier eine Intarsienimitation beabsichtigt. Die schwarzen Maureskenmotive sollten Einlagen in Ebenholz vortäuschen, während die roten, stets variierten Bänder das mit Drachenblut eingefärbte Holz Mahagoni vorspiegeln sollte.

Wohl in den zwanziger Jahren des 17. Jahrhunderts ist eine besonders qualitätvolle Deckenmalerei im ersten Dachgeschoss des stattlichen Bürgerhauses
Scheffelstraße 1 entstanden. Auf vier langen, aus schmalen Brettern zusammengesetzten Feldern befinden sich jeweils drei beziehungsweise einmal zwei große, reich und jeweils differenziert gemalte Beschlagwerkkartuschen, in die verschiedenartige Masken eingebunden sind. Vornehmlich in den oberen und unteren Bereichen sprießt grünes Blattwerk mit roten Früchten hervor. Innerhalb der Kartuschen befinden sich stets variierte Früchtebündel, umgeben von grünem Blattwerk. Sie bestehen aus Äpfeln, Birnen und Weintrauben, zu denen sich auch Möhren und kleine Kürbisse gesellen. Die mittlere Kartuschenreihe ist besonders hervorgehoben, indem hier frei und locker gemalte menschliche Gestalten, jeweils Mann und Frau, links und rechts das Beschlagwerk begleiten. Die Unterkörper von drei Paaren laufen nach unten lappig aus, während eines in voller Gestalt wiedergegeben ist. Weil in der Mitte ein Baum mit roten Äpfeln wächst und die Frau zu einem greift, könnte hier eine Anspielung auf den Sündenfall gegeben sein, worauf zusätzlich der Totenschädel unterhalb des Baumstammes deuten kann.

In Torgau zeigt jedoch auch ein Beispiel, dass nicht nur Häuser wohlhabender und gebildeter Besitzer individuell mit Wandmalereien ausgestattet wurden, sondern auch ein sehr kleines, einfaches, gar armseliges Handwerkerhaus Am Bäckerwall, heute am Rosa-Luxemburg-Platz 4. Es weist ebenfalls Wandmalereien aus der ersten Hälfte des 17. Jahrhunderts auf. ${ }^{37}$ Als Beispiel des Wohnens und Arbeitens ärmster Torgauer Bürger steht es im krassen Gegensatz zu dem reichen und prächtigen Bürgermeister-Ringenhain-Haus in der Breiten Straße 9. Denkmale der armen Bevölkerung sind überaus selten überliefert, umso wertvoller ist die Rekonstruktion des winzigen Handwerkerhauses, das im 17. Jahrhundert von Böttgermeistern und Schornsteinfegern bewohnt wurde.

Eine Besonderheit im Innern des Handwerkerhauses stellen kleine, an die Beilinien des Fachwerks angehängte schwarze und rottonige, meist glockenförmige Blüten dar. Aus der Ecke des Fachwerks wächst auch eine große runde Blüte. Sie stammen wohl aus der Zeit um 1620. Einmalig in Torgau ist die Darstellung eines hochseetauglichen, mit Kanonen bestückten dreimastigen Schiffes, in dem auch Matrosen erkennbar sind, in der oberen Wohnstube. Seine im Wind flatternden Segel zeigen Sterne. Mit Sternen verziert ist auch das die Wand abschließende Feld. Außerdem sind links Reste von zwei Türmen erhalten, die wohl zu einer ehemaligen Stadtlandschaft im Hintergrund des Schiffes gehören.

Vergleichbare Schiffsdarstellungen haben sich an der Decke im ersten Obergeschoss des Pirnaer Hauses Markt 14 erhalten. Sie stehen möglicherweise im Zusammenhang mit einer Reise des Kaisers Matthias im Sommer 1617 über Pirna nach Dresden. $^{38}$

Insgesamt spiegeln die in Torgau erhaltenen Malereien vom Ende des 15. bis zum Anfang des 17. Jahrhunderts eine hohe Kultur anspruchsvoller und auch einfacher bürgerlicher Auftraggeber wider. 